\title{
The Relationship between Epicardial Fat Thickness and Dampness-Phlegm Pattern in the Patients with ischemic stroke
}

\author{
Ji Myung Woo, Woo-Sang Jung, Seungwon Kwon, Seung-Yeon Cho, Seong-Uk Park, \\ Sang-Kwan Moon, Jung-Mi Park, Chang-Nam Ko, Ki-Ho Cho \\ Department of Cardiology and Neurology College of Korean Medicine Kyung Hee University
}

\begin{abstract}
Objectives: Epicardial fat is true visceral fat that is known to be associated with metabolic syndrome, high abdominal fat, insulin resistance, coronary artery diseases, low coronary flow reserve and subclinical atherosclerosis. Dampness-Phlegm pattern is one of the pattern diagnosis of traditional Korean medicine. Previous studies showed that Dampness-Phlegm pattern is associated with hypertension, dyslipidemia, metabolic syndrome. This study is intended to find association between Dampness-Phlegm pattern and epicardial fat thickness.

Methods: This study was a community-based single center trial. Ischemic stroke patients within 30 days after their ictus were enrolled. Epicardial fat thickness was measured using transthoracic echocardiography. Other measured and obtained variables are medical history, weight, height, body mass index, fasting blood glucose, cholesterol, triglycerol, high density lipoprotein, lipid and low density lipoprotein.

Results: Three hundred sixty six were enlisted, and one hundred forty were diagnosed with the Dampness-Phlegm pattern. Dampness-Phlegm pattern group had significantly thicker epicardial fat. Binary logistic regression also showed statistically significant result.

Conclusions: This study showed close association between epicardial fat and Dampness-Phlegm pattern. This result suggests a clue to standardization of pattern identification.
\end{abstract}

$\overline{\text { Key Words }}$ : Dampness-Phlegm pattern, Epicardial fat, Cerebral infarction

\section{Introduction}

Epicardial fat is true visceral fat of the heart. It is commonly found in the atrioventricular and interventricular grooves ${ }^{1)}$. Under normal condition, epicardial fat functions as a buffer that absorbs fatty acids and protection against high fatty acid levels of heart. It sometimes works as energy source to myocardium ${ }^{2}$. Also, it can protect myocardium from hypothermia. ${ }^{3)}$ However, evidence shows epicardial fat is a source of several proinflammatory and proatherogenic cytokines ${ }^{4)}$. During ischemia, oxidant -sensitive inflammatory signals in epicardial adipose tissue might lead to amplification of vascular inflammation and plaque instability due to its proximity. ${ }^{5)}$ Other studies have shown several associations between epicardial fat thickness and clinical and subclinical condition as follows; Metabolic syndrome, high abdominal fat, insulin resistance $^{6}$, coronary artery diseases ${ }^{7,9,9)}$, low coronary flow reserve ${ }^{10)}$ and subclinical atherosclerosis ${ }^{11)}$.

Korea has its own complementary and alternative

\footnotetext{
- Received : 28 November 2017

- Revised : 26 December 2017

- Accepted : 26 December 2017

- Correspondence to : Ji Myung Woo

KyungHeeDaeRo 23, KyungHee University Korean Medicine Hospital, Dongdaemun-Gu

Tel : +82-10-6662-0324, Fax : +82-2-958-9128, E-mail : dnwlaud@gmail.com
} 
medical practice called TKM (Traditional Korean Medicine). TKM has its own pathology, diagnosis and treatment. Pattern identification is one of diagnostic system of TKM that involves examination of body systematically to identify the pattern. A previous study standardized pattern identification for stroke patients ${ }^{12}$. One of the patterns is the Dampness-Phlegm pattern. The Dampness-Phlegm pattern is characterized by heaviness sensation of the limbs, feeling of stuffiness in the chest, reduced food intake, stickiness of the mouth, white slimy tongue coating and slippery pulse ${ }^{13)}$. Some of clinically identified symptoms of the Dampness-Phlegm pattern are overweight, pale complexion, pale tongue and slippery pulse ${ }^{12)}$. Also, study showed connection between metabolic syndrome and Dampness-Phlegm pattern $^{14)}$. These signs and symptoms gave clue to the pattern identification process. However, this process could vary among the clinicians. Therefore, quantifiable standard for the identification was needed.

This study was designed to find association between Dampness-Phlegm pattern and epicardial fat thickness, since both were associated with overweight and metabolic syndrome. Through this effort, epicardial fat thickness is expected to help standardizing Dampness-Phlegm pattern identification.

\section{Methods}

\section{Subjects}

This study was a community-based single center trial. Ischemic stroke subjects within 30 days after their ictus were enrolled from Kyung Hee University Korean Medicine Hospital (Seoul) from April 2007 to May 2013. Informed consent of all the study patients was obtained after a thorough explanation of the details. (KOMCGIRB-2011-02)
Case Report Form (CRF) and the Standard Operation Procedures (SOPs) were used. These were developed by the Experts Committee organized by the Korean Institute of Korean Medicine ${ }^{15}$. These included general patient information such as diagnosis and medical history. Weight and height were measured while patient wore only undergarments and hospital outfits. Blood samples were obtained during fasting, and levels of plasma glucose, cholesterol, triglycerol, high density lipoprotein (HDL), lipid, low density lipoprotein (LDL) were measured.

\section{Determination of Pattern-Identification}

The subjects were identified as having Dampness -Phlegm pattern when two independent traditional Korean medical doctors agreed. Criteria for diagnosis of dampness-phlegm pattern were based on suggestion by the Korean Institute of Oriental Medicine ${ }^{16)}$. When two doctors identified the pattern as the Dampness-Phlegm pattern, the patients were included in the Dampness-Phlegm pattern group. When both doctors identified the pattern other than the Dampness-Phlegm pattern, the patients were included in non-Dampness-Phlegm pattern group.

\section{Echocardiography}

The M-mode echocardiogram was performed with using $5 \mathrm{MHz}$ phased array with GE Vivid9(USA) or with Siemens Acuson SC2000(Germany). Echocardiogram was performed by doctors in cardiovascular center at Kyung Hee University Medical center. Epicardial fat was measured on the free wall of the right ventricle from the parasternal long-axis views. Epicardial fat was identified as an echo-free space in the pericardial layers. Aortic annulus was the reference of the measurement ${ }^{6}$. The entire measurement was by the author JM.

\section{Statistics}

\section{Measured Variables}


To determine baseline difference between the groups, t-test and chi-square test were used. P-value of less than 0.05 was considered statistically significant.

To estimate the odds ratio of Dampness-Phlegm pattern associated with epicardial fat thickness and other particular medical history, adjusted odds ratio (aOR) and 95 percent confidence intervals $(95 \% \mathrm{CI})$ were presented. Statistical analysis of adjusted odds ratio was done by multiple logistic regression. By multiple logistic regression, confidence interval, $\beta$ coefficient and $\mathrm{p}$-value for each factor were determined. Values that had $p$-value of less than 0.05 from table 1 were initially used for independent values. Independent values were either binary or continuous values. Binary values were number of male subjects, history of hypertension, diabetes mellitus. Continuous values were age, BMI, weight, total cholesterol, triglyceride, total lipid, LDL, fasting blood serum and epicardial fat thickness. Backward method was used. All the values were tested for multicollinearity. Values that showed multicollinearity were excluded from statistic. The entire analysis was performed using SPSS for Windows, version 12.0 (SPSS Inc., Chicago, Illinois, USA)

\section{Results}

\section{Clinical and laboratory characteristics of the study population}

Echocardiographic epicardial fat data could be obtained from three hundred sixty six patients. One hundred forty patients were diagnosed as the Dampness-Phlegm pattern(DP) and two hundred twenty six patients were as non-Dampness-Phlegm pattern (NDP). There were significant differences of weight, BMI, cholesterol, triglycerol, lipid, fasting serum glucose, LDL, sex, hypertension history, diabetes mellitus history and epicardial fat
thickness(Table 1). Subjects in DP group had significantly higher BMI, weight, higher number of hypertension and diabetes mellitus than NDP group. $(p<0.05)$.

\section{Epicardial fat thickness}

Epicardial fat thickness ranged from 0 through 16 $\mathrm{mm}$. The mean (SD) and median values of epicardial fat thickness were 5.35 (2.87) and 4.70. Mean (SD) of Epicardial fat thickness among the Dampness -Phlegm group was 7.30(2.85) and non-Dampness -Phlegm group was 4.14(2.13). There was significant difference in epicardial fat thickness between the two groups (Table 1).

\section{Multiple logistic regression and adjusted odds ratio}

Epicardial fat showed aOR value of 1.608 with 1.422 to $1.81895 \%$ confidence interval. Other significant values were number of male subjects (aOR : 0.310, 95\% CI: 0.159-0.606), weight (aOR: $1.063,95 \%$ CI: $1.025-1.101)$ and triglyceride (aOR: 1.005, 95\% CI: 1.001-1.009).

\section{Discussion}

Pattern identification in traditional Korean medicine is crucial in clinical practice. Through pattern identification, traditional Korean medical clinicians can evaluate patient and choose adequate treatment methods. Pattern identification can be done by obtaining various clues from patients. These clues include inspection, tongue diagnosis, pulse diagnosis and patients' symptoms. However, there is a problem in pattern identification. Clues gathered from the patients are mostly subjective and vary among different clinicians. Measurable standard for pattern identification is needed for establishing standardized traditional Korean medical practice.

The Dampness-Phlegm pattern is characterized by 
Table 1. Baseline Characteristic of Dampness-Phlegm Group and Non-Dampness-Phlegm Group

\begin{tabular}{|c|c|c|c|}
\hline Variable & $\mathrm{DP}(\mathrm{N}=140)$ & $\mathrm{NDP}(\mathrm{N}=226)$ & $P$ value \\
\hline Age, $y r$ & $65.4(10.6)$ & $67.4(10.3)$ & 0.069 \\
\hline Men, $n(\%)$ & $66(47.1)$ & $139(61.5)$ & 0.009 \\
\hline BMI $\left(\mathrm{kg} / \mathrm{m}^{2}\right)$ & $25.0(2.8)$ & $23.3(2.9)$ & $<0.001$ \\
\hline Weight $(\mathrm{kg})$ & $65.1(9.6)$ & $61.6(9.6)$ & 0.001 \\
\hline Height (cm) & $161.2(7.2)$ & $162.2(8.4)$ & 0.260 \\
\hline \multicolumn{4}{|l|}{ Medical History, $n(\%)$} \\
\hline Hypertension & $108(77.1)$ & $145(64.1)$ & 0.010 \\
\hline Diabetes mellitus & $54(38.6)$ & $62(27.4)$ & 0.028 \\
\hline Ischemic heart disease & $7(5)$ & $16(7.1)$ & 0.510 \\
\hline Ischemic stroke & $35(25)$ & $66(29.2)$ & 0.402 \\
\hline Total cholesterol (mg/dL) & $190.6(51.1)$ & $174.0(40.3)$ & 0.001 \\
\hline Triglyceride (mg/dL) & $155.2(89.3)$ & $121.3(65.9)$ & $<0.001$ \\
\hline Total lipid (mg/dL) & $593.1(156.10)$ & $532.1(114.0)$ & $<0.001$ \\
\hline LDL (mg/dL) & $115.9(44.3)$ & $106.2(37.6)$ & 0.029 \\
\hline HDL (mg/dL) & $43.2(10.3)$ & $43.7(11.4)$ & 0.658 \\
\hline Fasting blood serum glucose (mg/dL) & $120.3(42.9)$ & $110.4(39.3)$ & 0.027 \\
\hline Epicardial fat (mm) & $7.30(2.9)$ & $4.14(2.1)$ & $<0.001$ \\
\hline \multicolumn{4}{|c|}{$\begin{array}{l}\text { Valueside parenthesis are mean, and ion.Values outside parenthesis are means. Values inside parenthesis are standard deviation. } \\
\text { DP : Dampness-Phlegm pattern } \\
\text { NDP : non-Dampness-Phlegm pattern } \\
\text { BMl : Body Mass Index } \\
\text { HDL : High Density Lipoprotein }\end{array}$} \\
\hline
\end{tabular}

Table 2. Epicardial Fat Thickness and Dampness-Phlegm Pattern Correlation

\begin{tabular}{lcccc}
\hline & $\mathrm{PD}(\mathrm{N}=140)$ & $\mathrm{NPD}(\mathrm{N}=226)$ & $\mathrm{aOR}^{*}$ & $95 \% \mathrm{CI}$ \\
\hline Epicardial Fat $(\mathrm{mm})$ & $7.30 \pm 2.9$ & $4.14 \pm 2.1$ & 1.608 & $1.422-1.818$ \\
Age & $65.4 \pm 10.6$ & $67.4 \pm 10.3$ & 0.972 & $0.944-1.001$ \\
Men, $\mathrm{n}(\%)$ & $66(47.1)$ & $139(61.5)$ & 0.310 & $0.159-0.606$ \\
Weight $(\mathrm{kg})$ & $65.1(9.6)$ & $61.6(9.6)$ & 1.063 & $1.025-1.101$ \\
Triglyceride (mg/dL) & $155.2(89.3)$ & $121.3(65.9)$ & 1.005 & $1.001-1.009$ \\
\hline
\end{tabular}

* aOR (adjusted odds ratio) was adjusted for age, sex, BM((body mass index), weight, hypertension, diabetes mellitus, total cholesterol, triglyceride, total lipid, low density lipoprotein, fasting blood serum and epicardial fat thickness

fn the present study, odds rationt in ormation will be needed to achieve this goal. ndrome, this study is $\mathrm{pl}$

heaviness sensation of the limbs, feeling of stuffiness in the chest, reduced food intake, stickiness of the mouth, white slimy tongue coating and slippery pulse $^{13}$. Due to its heavy and slow properties, traditional Korean medical context often referred Dampness-Phlegm as a cause of obesity ${ }^{17}$. It is also closely related to excessive and inappropriate intake habit ${ }^{14}$ Since many medical conditions like obesity, diabetes mellitus and metabolic syndrome in modern society are in part associated with excessive intake,
Dampness-Phlegm pattern is a key to understanding these conditions.

Recent research showed that one of an indicator associated with conditions such as metabolic syndrome is epicardial fat thickness. Research reported cutoff values of epicardial fat thickness for particular conditions. Iacobellis et $\mathrm{al}^{6}$ suggested cutoff values of epicardial fat thickness of European men and women for metabolic syndrome are $\geq 9.5$ and $\geq 7.5$ respectively. Other values are high 
abdominal fat $(\geq 9.5$ and $\geq 7.5)$, extremely high abdominal fat ( $\geq 13$ and 10), insulin resistance $(\geq 9.5$ and $\geq 9.5)$ and high insulin resistance $(\geq 11$ and $\geq$ 11).

Epicardial fat and Dampness-Phlegm pattern both share an association with metabolic syndrome and other closely related medical conditions. Finding statistical association between two will deepen the understanding of these conditions.

This study is the first study to investigate association between the Dampness-Phlegm pattern and epicardial fat thickness. Subjects in the Dampness-Phlegm pattern group had significantly more female participants, higher BMI, weight, higher number of hypertension and diabetes mellitus, higher total cholesterol, triglyceride, total lipid, LDL and fasting blood serum glucose. $(p<0.05)$. Subjects in the Dampness-Phlegm pattern group also had significantly thicker epicardial fat. $(p<0.05)$.

In the present study, adjusted odds ratio was statistically analyzed using binary logistic regression.

Due to heterogeneity of two groups, binary logistic regression was needed to adjust interaction effects.

Difference in number of each gender group seems to be a coincidence during recruiting participants. Other baseline differences corresponded with the previous studies. The previous studies showed that the Dampness-Phlegm pattern is positively correlated to hypertension, diabetes mellitus, dyslipidemia ${ }^{17)}$ and metabolic syndrome ${ }^{14)}$.

Epicardial fat thickness was statistically related to Dampness-Phlegm pattern. Due to heterogeneity of the groups, adjusted odds ratio was used. The result was significantly different with odds ratio of 1.651 .

Limitation of this study is that it only included ischemic stroke patient. Since epicardial fat is correlated with coronary artery disease and metabolic syndrome, subjects with these conditions could also be included. Further study with different population will be necessary.

There are two strengths in this study. First, this study showed association between Dampness-Phlegm pattern and epicardial fat thickness with measurable value. Since there was a lack of measurable value to support Dampness-Phlegm pattern, this finding can help establish more standardized diagnostic system. Secondly, this study showed additional association between Dampness-Phlegm pattern and metabolic syndrome. Although there were few studies that showed the association before, this study reinforced the association between Dampness-Phlegm pattern and metabolic syndrome.

\section{Conclusion}

This study was to find association between Dampness-Phlegm pattern and Epicardial fat thickness. The conclusions are as follows:

1. Subjects in the Dampness-Phlegm pattern group had significantly more female participants, higher BMI, weight, higher number of hypertension and diabetes mellitus, higher total cholesterol, triglyceride, total lipid, LDL, fasting blood serum glucose and thicker epicardial fat thickness. $(p<0.05)$.

2. After Binary logistic regression, compared them in the non Damp Phlegm group. Age, BMI, the presence of hypertension and epicardial fat thickness between two groups were higher in the Dampness-Phlegm then in the no Dampness -Phlegm group.

Epicardial fat thickness is a measurable value that helps identifying Dampness-Phlegm pattern. This study can be a clue to pathophysiology of Dampness -Phlegm pattern. More study on epicardial fat and its formation will be needed to achieve this goal.

\section{Reference}

1. Iacobellis G, Corradi D, Sharma AM. Epicardial adipose tissue: anatomic, biomolecular and clinical relationships with the heart. National 
Clinical Practice Cardiovascular Medicine. 2005; 2:536-43.

2. Rabkin SW. Epicardial fat: properties, function and relationship to obesity. Obesity Reviews. 2007;8(3):253-61.

3. Sacks HS, Fain JN, Holman B, Cheema P, Chary A, Parks F, et al. Uncoupling protein-1 and related mRNAs in human epicardial and other adipose tissues: epicardial fat functioning as brown fat. Journal of Clinical Endocrinology and Metabolism. 2009;94:3611-5.

4. Baker AR, Silva NF, Quinn DW, Harte AL, Pagano D, Bonser RS, et al. Human epicardial adipose tissue expresses a pathogenic profile of adipocytokines in patients with cardiovascular disease. Cardiovascular Diabetology. 2006;13(5):1.

5. Mazurek T, Zhang L, Zalewski A, Mannion JD, Diehl JT, Arafat H, et al. Human epicardial adipose tissue is a source of inflammatory mediators. Circulation. 2003;108:2460-6.

6. Iacobellis G, Willens HJ, Barbaro G, Sharma AM. Threshold values of highrisk echocardiographic epicardial fat thickness. Obesity (Silver Spring). 2008;16:887-92.

7. Jeong JW, Jeong MH, Yun KH, Oh SK, Park EM, Kim YK. Echocardiographic epicardial fat thickness and coronary artery disease. Circulation Journal. 2007;71:536-9.

8. Saura D, Oliva MJ, Rodri'guez D, Pascual-Figal DA, Hurtado JA, Pinar E, et al. Reproducibility of echocardiographic measurements of epicardial fat thickness. International Journal of Cardiology. 2010;141(3):311-3.

9. Sarin S, Wenger C, Marwaha A, Qureshi A, Go $\mathrm{BD}$, Woomert CA, et al. Clinical significance of epicardial fat measured using cardiac multislice computed tomography. Journal of Cardiology. 2008;102:767-71.

10. Sade LE, Eroglu S, Bozbasx H, Ozbic,er S, Hayran M, Haberal A, et al. Relation between epicardial fat thickness and coronary flow reserve in women with chest pain and angiographically normal coronary arteries. Atherosclerosis. 2009;204:580-5.

11. Natale F, Tedesco MA, Mocerino R, de Simone V, Di Marco GM, Aronne L, et al. Visceral adiposity and arterial stiffness: echocardiographic epicardial fat thickness reflects, better than waist circumference, carotid arterial stiffness in a large population of hypertensives. European Journal of Echocardiogr. 2009;10:549-55.

12. HJ Kim, HS Bae, SU Park, SK Moon, JM Park, WS Jung. Clinical Approach to the Standardization of Oriental Medical Diagnostic Pattern identification in Stroke Patients. Evidence-Based Complementary and Alternative Medicine. 2011:7

13. World Health Organization. WHO international standard terminologies on traditional medicine in the western pacific region. $1^{\text {st }}$ edition. World Health Organization. 2007:120

14. IK Min, CH Kim, JW Hwang, JY Park, SY Lee, WW Choi et al. The Relation of Dampness -Phlegm and Metabolic Syndrome in Acute Stroke Patients. Journal of Korean Oriental Medicine. 2009;30(1):109-19.

15. Ko SG, Jun CY, Park JH, Ko HY, Yoon YS, Choi SM et al. Development of CRF and SOPs for the identification of risk factors of the cerebrovascular diseases in the east and west medicine. Korean Journal of Oriental Medicine. 2006;27:204-219.

16. Lee MG, Kang BK, Kim YB, Ko HY, Choi $\mathrm{SM}$, Seol IC et al. Improvement of tentative Korean standard differentiation of the symptoms and signs for stroke for clinical application. Korean Journal of Oriental Physiology \& Pathology. 2007;21:347-351.

17. Kang JS,KimDH, Shin HS, et al. The Study on Relationship of Dampness-Phlegm Tongue Diagnosis to hyperlipidemia in Stroke Patients. Journal of Korean Medicine Diagnostics. 2009; 13(2):24-33. 